EPJ Web of Conferences 88, 00021 (2015)

DOI: $10.1051 /$ epjconf/ 20158800021

(C) Owned by the authors, published by EDP Sciences - SIF, 2015

\title{
Symmetry energy dependence of light fragment production in heavy-ion collisions
}

\author{
H. H. Wolter ${ }^{1}$, M. Zielinska-Pfabe ${ }^{2}, \mathrm{P}$ DeCowski $^{2 \dagger}$ and \\ M. COLONNA ${ }^{3}$
}

${ }^{1}$ Fac. of Physics, University of Munich, D-85748 Garching, Germany

${ }^{2}$ Smith College, Northampton, MA 01063, USA

${ }^{3}$ INFN-Laboratori Nazionali del Sud, I-95123, Catania, Italy

\begin{abstract}
The pre-equilibrium light cluster emission in low to intermediate energy heavy ion collisions is a way to obtain information about the density and momentum dependence of the nuclear symmetry potential, i.e. about the stiffness of the symmetry energy and the neutron-proton effective mass splitting. We study the $n / p$ and $t /{ }^{3} H e$ ratios as a function of the energy of the emitted particles and find that these allow to disentangle these two aspects of the symmetry energy. The $t /{ }^{3} \mathrm{He}$ ratios are found to carry similar information as the $n / p$ ratios, making this a promising option for experimental investigations. More $n$-rich systems enhance the sensitivity to the symmetry energy, while double ratios between $n$-rich and $n$-poor systems tend to reduce it.
\end{abstract}

The density dependence of the nuclear symmetry energy is a topic of considerable interest in nuclear physics with important implications in astrophysics, particularly in the properties of neutron stars. Microscopic calculations of the symmetry energy show large differences, especially at supersaturation densities, due to uncertainities of the short range behavior of the isovector forces. Thus it is of interest to investigate the symmetry energy via nuclear structure observables and in heavy ion collisions. The advantage of the latter is that by choosing different collision systems, incident energies and centralities one can access different densities and asymmetries of

\footnotetext{
${ }^{\dagger}$ deceased
} 
nuclear matter and study the explicitly the momentum dependence of the potentials. It is customary to refer to a density dependence of the symmetry energy with a large slope at saturation as asy-stiff, and to the opposite situation as asy-soft.

In this contribution we consider nuclear matter around and below saturation densities in collision with energies from the Fermi energy to a few $100 \mathrm{MeV}$ per particle, where the decomposition into fragments with the emission of light clusters is the main decay mode, which allows to obtain information on the properties of the nuclear symmetry energy. A number of observables have been identified as sensitive to the nuclear symmetry energy. One is the pre-equilibrium emission of nucleons and light fragments in the initial stages of the collision, which depends directly on the neutron and proton potentials. To enhance the sensitivity to the nuclear symmetry energy one considers ratios or differences of observables of isobaric pairs of particles, such as yields of neutrons to protons, tritons to ${ }^{3} H e$, or $\pi^{-}$to $\pi^{+}$, or similarly for flow observables.

The most direct effect of the symmetry energy is expected to be seen in the neutron to proton ratios. For a $n$-rich medium the neutron potential is repulsive below saturation, and the repulsion is stronger for an asy-soft symmetry energy. Therefore the neutron over proton ratio should be larger for an asy-soft symmetry energy. In fact, this has been observed in medium energy collisions of different $\mathrm{Sn}$ isotopes [1]. An experimental difficulty is that neutron and protons are measured with different phase space coverage. To reduce the corresponding uncertainties the ratios of the neutron-to-proton ratios between systems of different asymmetry, so-called double ratios, were considered in ref. [1]. However, in double ratios some of the sensitivity to the detailed properties of the symmetry energy may be lost as will be seen below. It is therefore of interest to consider ratios of light charged particles, in particular $t /{ }^{3} \mathrm{He}$, since the experimental uncertainties should be reduced.

The effect of the momentum dependence of the symmetry energy on $n / p$ ratios was noted some time ago in ref. [2]. A more systematic study considering both the density and momentum dependence was performed by Rizzo et al. [3] for $S n+S n$ systems for energies between 50 and 100 $\mathrm{AMeV}$. At higher energies of 400 to $600 \mathrm{AMeV}$ the $n / p$ ratio, but also the $t /{ }^{3} \mathrm{He}$ ratios were studies by Giordano et al., [4] and Feng [5], where it was seen that the momentum dependence provided the dominating effect on these ratios. At these energies no data exist, yet. The present study is directed to a systematic theoretical study of the yield ratios, motivated by experimental data of the system ${ }^{136,124} \mathrm{Xe}+{ }^{124,112} \mathrm{Sn}$ at energies of $\mathrm{E} / \mathrm{A}=32$ to $150 \mathrm{MeV}$ [6], with a preliminary report in ref. [7]. A similar theoretical 
study again in the $S n+S n$ system has recently been performed by Zhang et al. [8].

The physical input into transport simulations of heavy ion collisions are the mean field potential and the in-medium coss section. In general, the mean field depends on density, but also on momentum, which arises from exchange and correlation contributions. This momentum dependence is important in heavy ion collisions where the particles may have high momenta, while it is only implicit in the equation-of-state, which is integrated over the momentum distribution of uniform nuclear matter. The momentum dependence of the isovector potential can be characteized as a function of density and momentum in terms of an effective mass

$$
\frac{m_{i}^{*}}{m}=\left(1+\frac{m}{\hbar^{2} k} \frac{\partial U_{i}}{\partial k}\right)^{-1} ; i=(n, p) .
$$

Thus a splitting of the effective masses of protons and neutrons appears. The magnitude and even the sign of this splitting is controversial in many-body calculations and in analyses of heavy ion collisions [9].

Here we present results of transport calculations for the $n$-rich system ${ }^{136} \mathrm{Xe}+{ }^{124} \mathrm{Sn}$ and the $n$-poor system ${ }^{124} \mathrm{Xe}+{ }^{112} \mathrm{Sn}$ at energies between 32 and $150 \mathrm{AMeV}$. The simulations are performed with the Stochastic Mean Field (SMF) simulation code of the BUU transport equations, which is an approximate implementation to a transport with a fluctuation term, i.e. the Boltzmann-Langevin equation [10]. The mean field is parametrized as a Gale-Bertsch-DasGupta type potential which allows to change the density and momentum dependence of the symmetry potential without changing the isoscalar behavior. We perform calculations with all four combinations of asy-soft (so) and -stiff $(s t)$ density dependence and ordering of the effective masses $(n): m_{n}^{*}>m_{p}^{*}$ and $p: m_{p}^{*}>m_{n}^{*}(p)$. Correspondingly the models are denoted as son, sop, stn, stp, respectively. Clusters are identified by coalescence at the freeze-out time, where we employ two methods: $(\mathrm{CO})$ coalescence in phase space with coalescence radii of $r_{0}=1.6 \mathrm{fm}$ and $p_{0}=1.3 \mathrm{fm}^{-1} ;(\mathrm{DC})$ density-cut in coordinate space with $\rho>0.03 \mathrm{fm}^{-3}$ as the "liquid" and everything else as the gas of neutrons and protrons. We consider central collisions with impact parameter of $b=2 \mathrm{fm}$.

In Fig. 1 we display the total yields per collision for the two reactions and the different light particles up to ${ }^{4} \mathrm{He}$ for a collision energy of $32 \mathrm{MeV} / \mathrm{A}$. Protons and neutrons are shown for both methods of fragment recognition: the phase space coalescence $(\mathrm{CO})$ and the density cut (DC) methods. The large difference between the two methods is related to the intermediate mass fragments (IMF) with $A>4$ (not shown here), which are heavier in the DC 


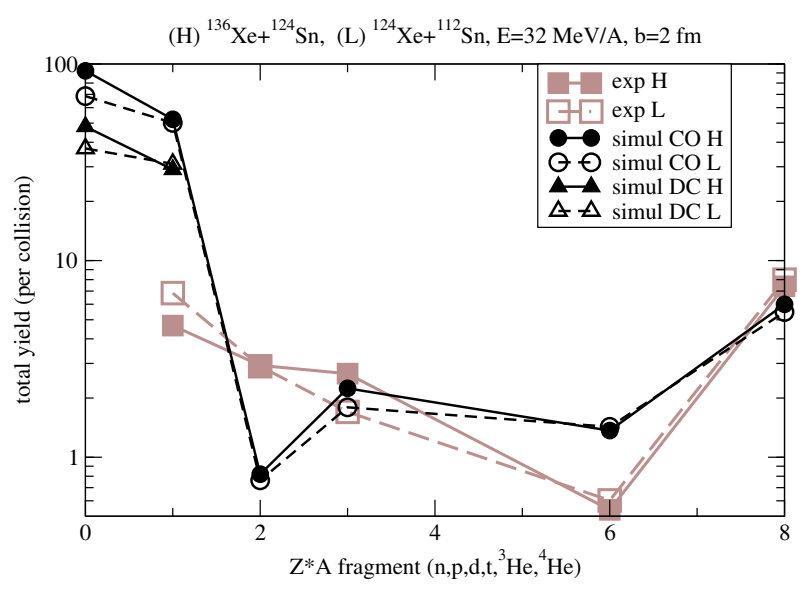

Figure 1: Total yield per collision of different light particles in collisions at 32 $\mathrm{MeV} / \mathrm{A}$ of ${ }^{136} \mathrm{Xe}+{ }^{124} \mathrm{Sn}$ and ${ }^{124} \mathrm{Xe}+{ }^{112} \mathrm{Sn}$, denoted as heavy (H) (full symbols and solid lines) and light (L) (open sympols and dashed lines), respectively. The different particles are plotted on the abcissa according to the product $Z * A$. The experimental data from ref. [6] are plotted in grey with squares. For the simulations (black) we distiguish fragment recognition by coalescence (circles, $\mathrm{CO}$ ) and by a density-cut criterion (triangles, DC). The simulations are shown for the son case $\left(\right.$ asy-soft, $\left.m_{n}^{*}>m_{p}^{*}\right)$.

method. We also show by grey squares the total yield of the data, which are obtained only for charged particles [6]. It is seen that protons are substantially overpredicted, showing that the clustering into IMFs is much stronger in the experiment. The pattern for the production of the other particles is roughly produced, with deuterons somewhat under- and ${ }^{3} H e$ 's overproduced. The yield of alpha particles is about correct, and is larger than that of the $\mathrm{A}=3$ particles, showing that the mean field begins to give some extra binding to alpha clusters. The experimental data include particles from the deexcitation of the primary fragments while the calculations do not. Thus this comparison is qualitative. It is evident, however, that generally the treatment of cluster production in transport calculations is still rather qualitative and needs to be improved. It is hoped that the ratios of light cluster yields, as studied here, are still meaningful, and, in particular, less sensitive to the evaporation component.

In Fig. 2 we show various single ratios for the different systems and incident energies in each case for the different models of the symmetry energy. The left and middle columns shows the $n / p$ ratios for the $n$-poor and $n$-rich systems, respectively, and the right column the $t /{ }^{3} \mathrm{He}$ ratio for the $n$-rich system. The upper row is for cm-energy of $32 \mathrm{AMeV}$, the lower one for 150 
$\mathrm{AMeV}$.
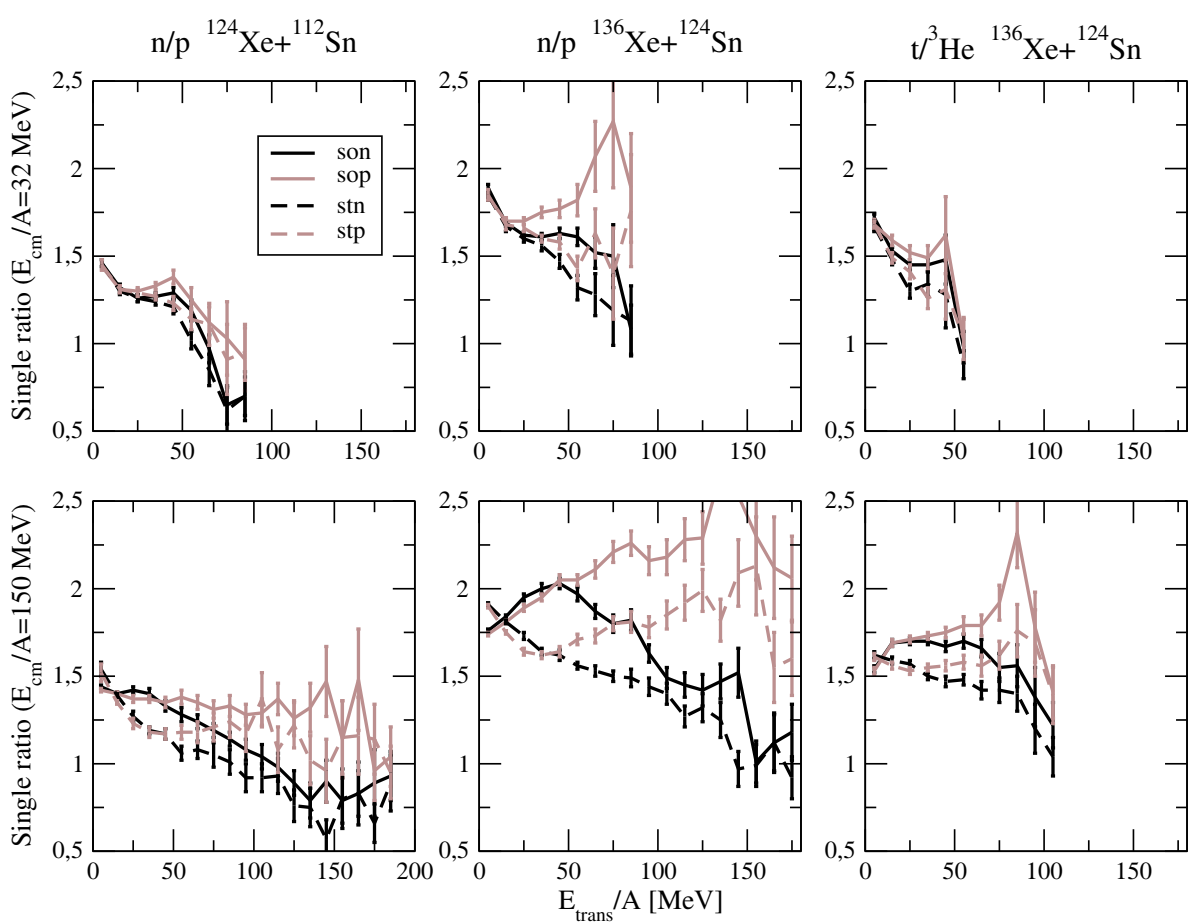

Figure 2: Single ratios of light emitted particles as a function of transverse energy per nucleon for the different models (see text): asy-soft solid, asy-stiff dashed, larger neutron effective mass black, larger proton effective mass grey. Left column $n / p$ single ratios for the $n$-poor, middle for the $n$-rich system and right column $t /{ }^{3} \mathrm{He}$ ratio for $n$-rich system. Upper row for incident energies of $32 \mathrm{AMeV}$, and lower row for $150 \mathrm{AMeV}$. Error bars represent statistical errors of the simulation.

It is seen that the difference between the models is more pronounced the higher the energy and the more $n$-rich the system. A very interesting behavior of these single ratios on the density (asy-soft or -stiff) and momentum $\left(m_{n}^{*}>m_{p}^{*}\right.$ or $\left.m_{p}^{*}>m_{n}^{*}\right)$ dependence is observed. It is most clearly seen in middle lower panel for the $n / p$ ratio in the $n$-rich system at the higher energy: The ratio at low transverse energies is dominated by the asy-stiffness of the symmetry energy, since the two models with an asy-soft dependence (solid lines) on one hand, and an asy-stiff dependence (dashed lines) on the other behave similarly. This is understood since the more repulsive neutron potential for asy-soft leads to a higher $n / p$ ratio. At high transverse momentum, on the other hand, the two models with $m_{p}^{*}>m_{n}^{*}$ (grey lines) and with $m_{n}^{*}>m_{p}^{*}$ (black lines), respectively, behave similarly. 
Now the particles with the smaller effective mass are emitted more readily. Similar observations were made in refs. $[3,8]$ where it was also shown that the crossing is related to the crossing of the neutron and proton potentials as a fuction of energy. It thus appears, that these ratios give the very interesting possibility to separate the density and the momentum behavior of the symmetry potential. This behavior is also seen in the $n$-poor system (left column), but less strongly as one would expect. At lower energy (upper row) the dependence on the stiffness is less ppronounced. In the right column we see that the corresponding $t /{ }^{3} \mathrm{He}$ ratios behave similarly as the $n / p$ ratios. This is not as clearly seen in the figure, since the $t$ and ${ }^{3} H e$ spectra are cut off at lower energies due to the statistics of the simulations.

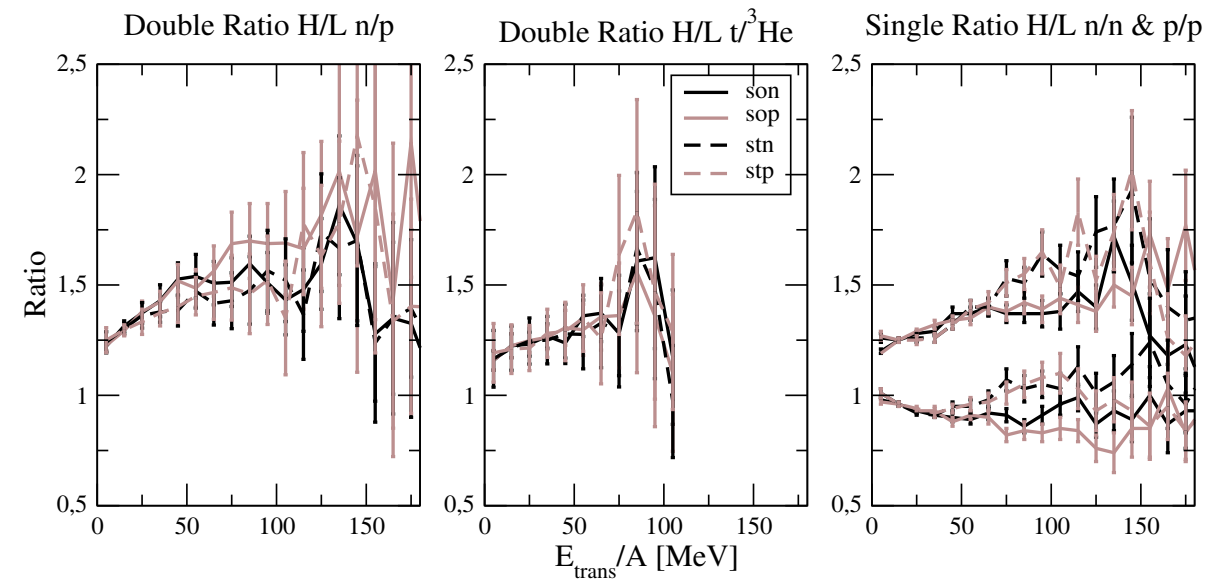

Figure 3: Double $n / p$ ratio (left panel) and $t /{ }^{3} \mathrm{He}$ ratio (middle panel) for the two systems $(\mathrm{H}$ and $\mathrm{L})$ at $150 \mathrm{AMeV}$ as a function of transverse energy. The right panel shows the $n$ (upper curves) and $p$ (lower curves) single ratios between the two systems. The meaning and signature of the curves is as in Fig. 2. Error bars are again statistical errors of the simulation.

Double ratios are shown in Fig. 3 at $150 \mathrm{AMeV}$ in the left panel for $n / p$ and in the middle panel for $t /{ }^{3} \mathrm{He}$. It is generally seen that the double ratios are considerably less sensitive to the models for the symmetry energy. The dependence on the stiffness at the lower transverse energies has practically disappeared and the dependence on the effective mass is much smaller. Thus in the double ratios a considerable part of the sensitivity to the characteristics of the symmetry energy is lost. The consideration of single ratios seems preferable, esp. when there is no strong experimental argument against it, as for the $t /{ }^{3} \mathrm{He}$ ratios. The right column shows the (single) ratios of neutrons (upper curves in this panel) and of protons (lower curves) for the $n$-rich over 
the $n$-poor system, which have also been suggested as an isospin-sensitive observable. These ratios essentially show only the dependence on the density dependence of the symmetry energy, particularly for the $n$ ratio, since the curves with an asy-stiff behavior (dashed lines) lie above the curves for the asy-soft dependence (solid lines) for all transverse energies. It appears that the effect of the momentum dependence cancels out to a large extent.

In summary, the present study shows that ratios of yields of isobaric pairs in pre-equilibrium cluster emission can be a useful tool to learn about the density and momentum dependence of the symmetry potential. In particular, the dependence of these ratios as a function of the transverse emission energy appears as a way to disentangle the density and momentum dependence. It is seen that $t /{ }^{3} \mathrm{He}$ ratios carry much the same information as $n / p$ ratios, and thus represent a promising experimental option. We also find, that double ratios tend to reduce the information on the detailed dependence of the nuclear symmetry energy. A more detailed comparison with existing and future experiments will be of great interest.

This work was supported in part by the Universe Cluster of Excellence of the Deutsche Forschungsgemeinschaft, Germany.

\section{References}

[1] M. Famiano, et al., Phys. Rev. Lett. 97, 052701 (2006)

[2] B.A. Li, et al., Nucl. Phys. A 735, 563 (2004); B.A. Li, et al., Phys. Lett. B 634, 378 (2006)

[3] J. Rizzo, M. Colonna, M. Di Toro, Phys. Rev. C 72, 064609 (2005)

[4] V. Giordano, et al., Phys. Rev. C 81, 044611 (2010)

[5] Z.Q. Feng, Phys. Lett B 707, 83 (2012); Nucl. Phys. A 878, 3 (2012)

[6] private communication

[7] H.H. Wolter, et al., EPJ Web of Conf. 66, 03097 (2014)

[8] Y.X. Zhang et al., arXiv: 1403:3790 [nucl-th]

[9] C. Fuchs, H.H. Wolter, Eur. Phys. Jour. A 30, 5 (2006); B.A. Li, X. Han, arXiv: 1304.3368 [nucl-th]

[10] V. Baran, et al., Phys. Rep. 410, 335 (2005) 